В. А. Халимончук ${ }^{1}$, А. В. Кучин ${ }^{1}$,

В. В. Токаревский ${ }^{2}$

${ }^{1}$ Государственный научно-технический центр по ядерной и радиационной безопасности, г. Киев, Украина

${ }^{2}$ Институт проблем Чернобыля, г. Киев, Украина

\section{Исследования нейтронно-} физических характеристик реактора РБМК-1000 Чернобыльской АЭС в Институте ядерных исследований АН УССР в до- и послеаварийный период: ретроспективный анализ

Рассмотрены вопросы научно-технического сотрудничества ИЯИ АН УССР $c$ Чернобыльской АЭС в до- и послеаварийный период. Выполнен ретроспективный анализ состояния и развития программного обеспечения для анализа безопасности РБМК-1000. Акцентируется внимание на необходимости внедрения в исследования безопасности наиболее современных программных средств и создания в Украине ведомственного научно-исследовательского института для решения всего спектра задач атомной энергетики.

Ключевые слова: реактор РБМК, паровой коэффициент реактивности, оперативный запас реактивности, концевой эффект реактивности, аксиальное распределение поля нейтронов, изменение реактивности, переходные процессы, эффективная доля запаздывающих нейтронов.

\section{В. А. Халімончук, О. В. Кучін, В. В. Токаревський}

Дослідження нейтронно-фізичних характеристик реактора РБМК-1000 Чорнобильської АЕС в Інституті ядерних досліджень АН УРСР в до- та післяаварійний період: ретроспективний аналіз

Розглянуто питання науково-технічної співпраці ІЯД АН УРСР з Чорнобильською AEC в до- та післяаварійний період. Виконано ретроспективний аналіз стану та розвитку програмного забезпечення для аналізу безпеки РБМК-1000. Акцентується увага на необхідності впровадження в дослідження безпеки найбільш сучасних програмних засобів та створення в Україні відомчого науково-дослідного інституту для вирішення всього спектру задач атомної енергетики.

Ключові слова: реактор РБМК, паровий коефіцієнт реактив ності, оперативний запас реактивності, кінцевий ефект реактивності, аксіальний розподіл поля нейтронів, змінення реактивності, перехідні процеси, ефективна частка запізнілих нейтронів.

(C) В. А. Халимончук, А. В. Кучин, В. В. Токаревский, 2016
$\mathrm{O}$ дним из направлений развития атомной энергетики СССР в 70-е годы прошлого столетия стало строительство АЭС с реактором РБМК1000 - прототипом промышленных реакторов по наработке оружейного плутония. Установленные мощности на АЭС в СССР в то время в основном увеличивались именно на реакторах данного типа исходя из того, что они не нуждались в таком крупногабаритном оборудовании, как корпус реактора и парогенератор, для производства которых достаточные мощности соответствующих заводов были ограничены. Первый блок Чернобыльской АЭС с РБМК-1000, введенный в эксплуатацию в 1977 году, являлся четвертым по счету реактором данного типа в СССР.

РБМК-1000 в значительной степени гетерогенная система в том смысле, что физические и материальные свойства активной зоны существенно неоднородны в пространстве. Это связано как с конструкцией органов регулирования, индивидуальным подводом теплоносителя к каждому технологическому каналу (ТК) и различным аксиальным распределением плотности теплоносителя в каждом ТK, так и с индивидуальным аксиальным распределением линейной мощности в технологических каналах. Это означает наличие пространственной неравномерности в поле температур графитовой кладки и топлива, концентрации ксенона, плотности теплоносителя, глубины выгорания топлива. Уже изначально было понятно, что такой реактор качественно можно рассчитывать только при использовании трехмерных расчетных кодов. Однако из-за отсутствия необходимого мощного парка вычислительной техники физический расчет реактора осуществлялся в двумерной геометрии с использованием двухгрупповой диффузионной модели переноса нейтронов, реализованной в программе «БОКР» Главного конструктора РБМК-1000. Результаты расчета пространственного распределения поля энерговыделения по БОКР, выполняемого на внешней ЭВМ (БЭСМ-6), использовались в качестве реперного поля энерговыделения для ввода в систему физического контроля распределения энерговыделения (СФКРЭ) и обеспечения пространственного контроля энерговыделения во всем объеме активной зоны в течение некоторого времени до ввода обновленного реперного расчета.

Для обеспечения научно-технической поддержки эксплуатации РБМК-1000 в 1975 году в ИЯИ АН УССР была создана группа расчетчиков (В. А. Халимончук, А. В. Кучин, В. Д. Марьяненко, И. Д. Орап), которая с начала 80-х годов работала в отделе атомной энергии под руководством доктора физико-математических наук В. В. Токаревского. К одной из основных задач этой группы, кроме исследования нейтронно-физических характеристик (НФХ) РБМК, относились выполнение и передача на Чернобыльскую АЭС реперных физических расчетов. На начальном этапе эксплуатации реактора РБМК-1000 результаты физического расчета по БОКР довольно неплохо согласовывались с экспериментальными показаниями СФКРЭ, однако в дальнейшем начали существенно отличаться от них из-за роста неопределенностей в данных по глубине выгорания топлива, плотности теплоносителя и другим физическим параметрам, которые в БОКР использовались в качестве исходных данных. Увеличение неопределенностей в исходных данных для физического расчета РБМК-1000 потребовало в дальнейшем разработки специальных алгоритмов восстановления поля энерговыделения на основе использования экспериментальных показаний тока датчиков СФКРЭ. 
Поведение пространственного поля энерговыделения в различных режимах эксплуатации РБМК в начале 80 -х годов изучалось на упрощенных моделях (одномерные и двумерные модели), что было сопряжено, в первую очередь, с отсутствием емких (по объему оперативной памяти и скорости счета) и стабильно работающих ЭВМ: по одномерной программе изучалось поведение аксиального распределения, а по двумерной программе - распределение в х-yгеометрии. Ряд исследований поведения НФХ выполнялся в трехмерной геометрии на модели полиячейки (повторяющийся фрагмент активной зоны) РБМК. Эти исследования велись в тесном сотрудничестве со специалистами ИАЭ им. И. В. Курчатова. В этот период на основе программного комплекса KVARK [1], разработанного в ИАЭ им. И. В. Курчатова и реализующего двухгрупповую модель реактора на основе уравнений Галанина-Фейнберга, была создана двумерная программа [2], а позже - трехмерная двухгрупповая диффузионная программа расчета полиячейки [3] для исследования переходных процессов в РБМК в адиабатическом приближении. Обе программы были применены для изучения радиальных и аксиальных деформаций поля энерговыделения в режимах изменения мощности реактора с учетом обратных связей по концентрации ядер ксенона, температуры графитовой кладки и мощностного коэффициента реактивности. Изменение плотности теплоносителя в [3] при изменении уровня мощности реактора учитывалось специально разработанной программой TEGA-2 [4]. С помощью программы [2] была разработана методика определения глубин погружения СУЗ для вывода реактора на МКУ мощности, минимизирующая радиальный коэффициент неравномерности энерговыделения при подъеме мощности реактора. На модели полиячейки реактора [3] исследованы причины возникновения перекосов поля нейтронов по высоте активной зоны при уменьшении уровня мощности реактора. Более детально результаты этих исследований обсуждаются далее в статье с использованием разработанной позже полномасштабной модели активной зоны.

Необходимость разработки полномасштабных трехмерных моделей для исследования НФХ РБМК и их прогнозирования стала очевидной в середине 80 -х годов. Уже к пуску четвертого энергоблока Чернобыльской АЭС появились две полномасштабные программы стационарного расчета РБМК-1000. Одна из них - TREP [5] - была реализована на одногрупповой диффузионной модели, вторая - DST [6] - на двухгрупповой диффузионной модели. Каждая из программ содержала блок восстановления поля энерговыделения по показаниям датчиков контроля энерговыделения СФКРЭ. Эти программы и используемые ими библиотеки нейтронно-физических констант отлаживались и тестировались на данных эксплуатации энергоблоков Чернобыльской АЭС и результатах экспериментов во время физических пусков. Для повышения скорости счета были проведены предварительные исследования по соответствующей оценке различных математических методов ускорения сходимости внутренних и внешних итераций в каждом из алгоритмов. В итоге программы были валидированы на данных эксплуатации блоков РБМК-1000 Чернобыльской АЭС в стационарных состояниях и реализованы на ЭВМ серии ЕС. С помощью программы DST [6] путем обсчета эксперимента по взвешиванию стержней СУЗ группы АР-2 (автоматического регулирования мощности) подтвердилась возможность ввода положительной реактивности при их погружении в активную зону с верхнего крайнего положения [7].
На основе разработанных блоков стационарного расчета программы [5, 6] были модернизированы для проведения расчетов медленных нестационарных процессов с использованием адиабатического подхода. С помощью [5] в дополнение к полиячеечным расчетам проведены исследования объемных деформаций поля энерговыделения на РБМК-1000 при снижении мощности реактора на полномасштабной модели.

Опыт эксплуатации РБМК-1000 показал, что в переходных процессах, связанных со снижением мощности энергоблока, наблюдаются значительные деформации плотности потока тепловых нейтронов по высоте активной зоны. Вследствие этого линейные нагрузки мощности на твэлы в переходном процессе снижения мощности могут иметь бульшие значения, чем в стационарном состоянии на номинальном уровне мощности, и даже превысить допустимые значения. Из анализа причин, вызывающих значительные высотные деформации плотности потока тепловых нейтронов в режимах глубокого снижения мощности реактора, следовало, что это связано с пространственным перераспределением НФХ активной зоны, влияющим на реактивность, и с выбранным способом компенсации реактивности. При переводе реактора на уровень мощности $50 \%$ номинальной происходит переотравление активной зоны ${ }^{135} \mathrm{Xe}$, изменяется температура графитовой кладки, регулируется расход теплоносителя. В результате меняется реактивность, компенсируемая стержнями регулирования. Если компенсация реактивности осуществляется извлечением из активной зоны частично погруженных стержней РР или ПКАЗ, высотные деформации достигают максимальных значений, На рис. 1 видно, что высотные деформации достигают максимальных значений к моменту времени 4,5 ч. Кривая 3 на этом рисунке, в отличие от кривой 2, учитывает регулирование расхода теплоносителя, т. е. предполагается, что приблизительно через 15 мин с начала переходного процесса устанавливается расход теплоносителя через активную зону, соответствующий мощности реактора $50 \% W_{\text {ном}}$. Поскольку при этом средний расход теплоносителя через технологический канал реактора уменьшается с 6,7 до 4,2 кг/с, аксиальное распределение плотности теплоносителя в переходном процессе с регулировкой расхода через реактор мало отличается

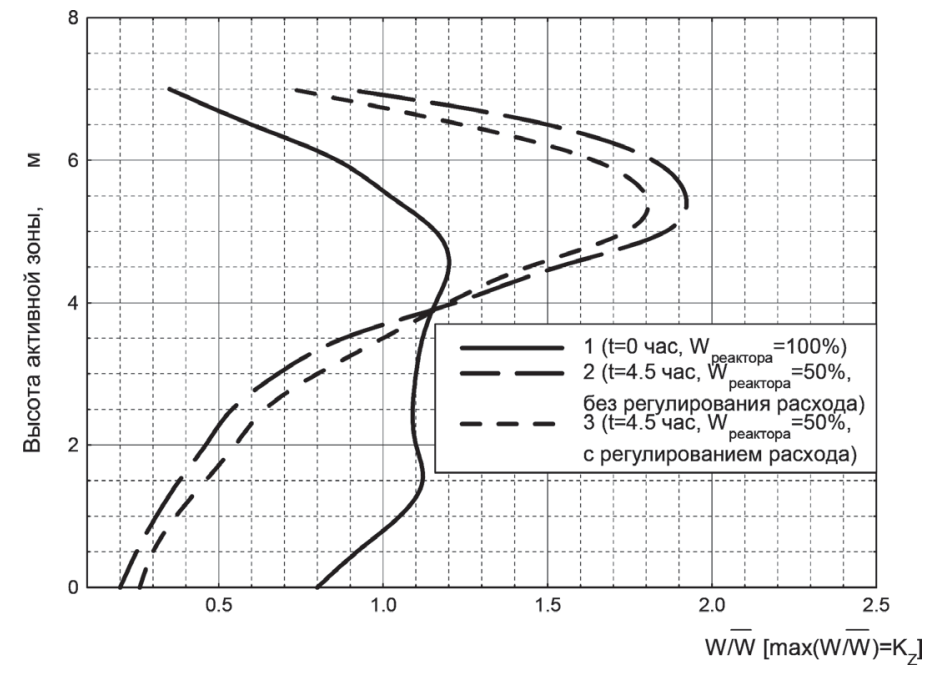

Рис. 1. Распределение энерговыделения по высоте реактора (компенсация реактивности стержнями РР и ПКАЗ) 
от начального распределения. Из анализа результатов расчета следует, что при компенсации реактивности стержнями РР и ПКАЗ, поглощающие части которых находились вверху активной зоны, максимум в распределении плотности потока тепловых нейтронов также смещается вверх. Это приводит к тому, что в верхней части активной зоны происходит более интенсивное выжигание ${ }^{135} \mathrm{Xе}$ и, как следствие, - дополнительное увеличение размножающих свойств, что способствует смещению максимума поля энерговыделения в верхнюю часть. В результате аксиальное распределение поля значительно искажается (см. рис. 1), и значение коэффициента неравномерности энерговыделения по высоте $\left(K_{Z}\right)$ достигает приблизительно 2 с максимумом вверху. Такое состояние сохраняется в течение нескольких часов, прежде чем образование ${ }^{135}$ Хе и ${ }^{135} \mathrm{I}$ не приведет к уменьшению размножающих свойств в районе максимума потока тепловых нейтронов. Сравнение кривых 2 и 3 на рис. 1 показывает, что учет регулирования расхода теплоносителя приводит к незначительному снижению максимума в распределении энерговыделения.

Расчетные исследования показали, что наименьшее искажение высотного распределения может быть достигнуто, если компенсацию отрицательной реактивности из-за отравления ${ }^{135} \mathrm{Xe}$ осуществлять одновременно стержнями УСП, РР и ПКАЗ (рис. 2). При этом, как и ранее (рис. 1), расход регулировался, но обратная связь по температуре графитовой кладки не учитывалась. Видно, что при компенсации реактивности одновременно стержнями УСП, РР и ПКАЗ увеличение максимума в распределении энерговыделения по высоте активной зоны незначительно. Объясняется это конструкцией УСП, которые, в отличие от стержней РР и ПКАЗ, вводятся в нижнюю часть активной зоны.

Учет обратной связи по температуре графитовой кладки приводит к увеличению искажения аксиального распределения поля энерговыделения. Физически это выражается в том, что при компенсации реактивности в переходных процессах снижения мощности путем извлечения стержней РР и ПКАЗ максимум в распределении плотности потока тепловых нейтронов, смещаясь вверх, приводит не только к увеличению выгорания ${ }^{135} \mathrm{Xe}$, но и повышению

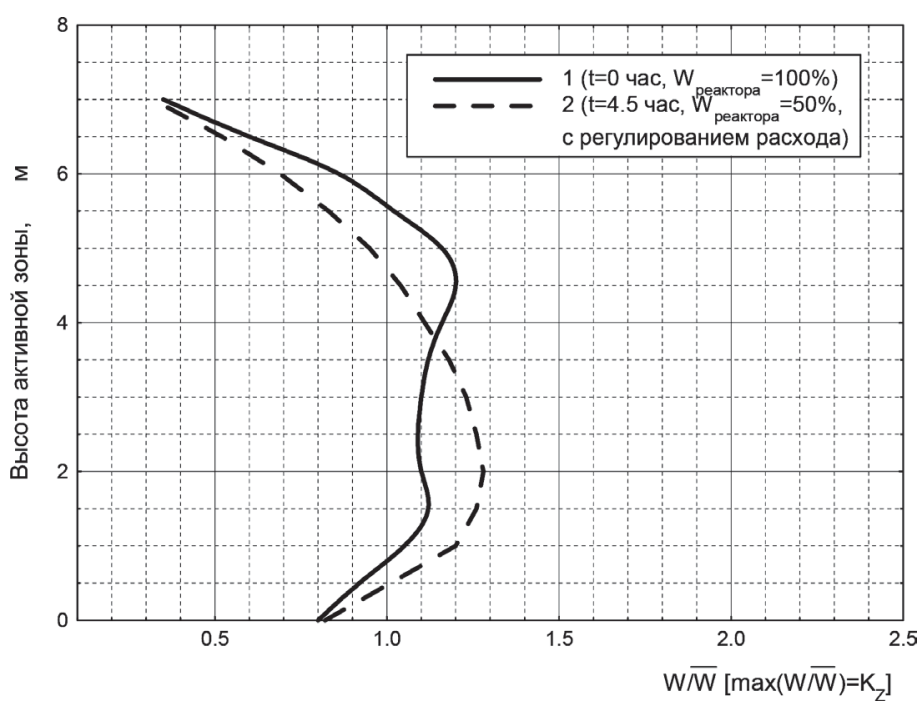

Рис. 2. Распределение энерговыделения по высоте реактора (компенсация реактивности стержнями УСП, РР и ПКАЗ)

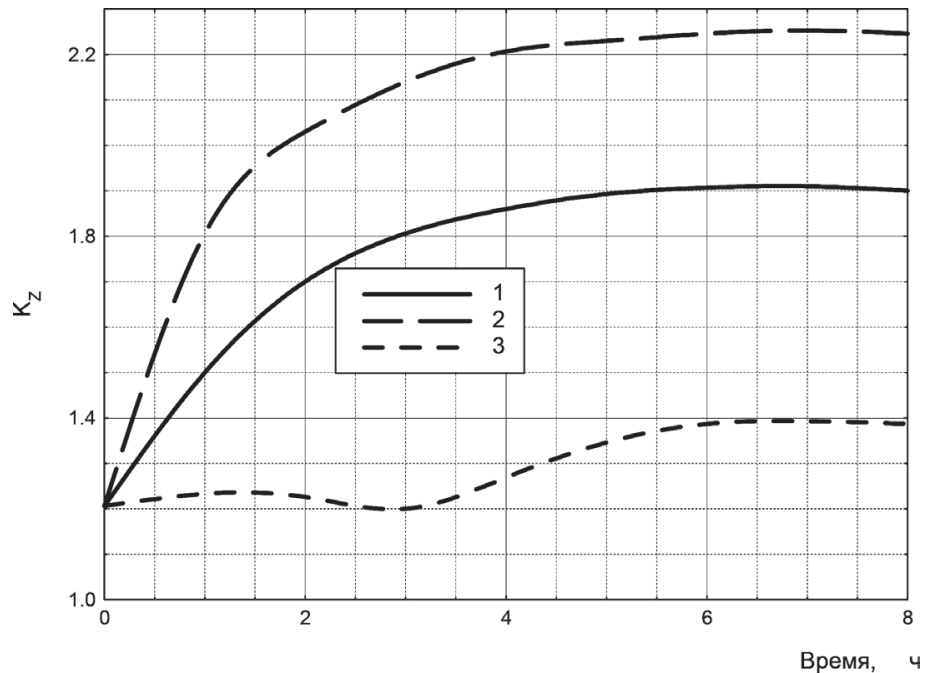

Рис. 3. Изменение коэффициента неравномерности энерговыделения по высоте активной зоны: 1 - компенсация реактивности стержнями РР, ПКАЗ; учет обратных связей по ${ }^{135} \mathrm{Xе} \mathrm{и} \mathrm{плотности} \mathrm{теплоносителя;} 2$ - компенсация реактивности стержнями РР, ПКАЗ; учет обратных связей по ${ }^{135} \mathrm{Xe}$, плотности теплоносителя и температуре графита; 3 - компенсация реактивности стержнями УСП, РР и ПКАЗ; учет обратных связей по ${ }^{135} \mathrm{Xe}$, плотности теплоносителя и температуре графита

температуры графита. Поэтому возрастание размножающих свойств в верхней части активной зоны усиливается по сравнению с действием только обратной связи по ${ }^{135} \mathrm{Xe}$, и коэффициент неравномерности энерговыделения по высоте активной зоны $\left(K_{Z}\right)$ возрастает (рис. 3, кривая 2). Во всех вариантах расчета, представленных на рис. 3, учитывалось уменьшение расхода теплоносителя через активную зону до величины, соответствующей 50 \%-му уровню мощности.

Таким образом, при компенсации реактивности стержнями РР или ПКАЗ аксиальные деформации наиболее значительны, коэффициент неравномерности энерговыделения по высоте активной зоны $\left(K_{Z}\right)$ достигает 2 . Поэтому на пониженных уровнях мощности реактора линейные нагрузки мощности на твэл могут превзойти допустимые. Для предотвращения подобного необходимо в переходном процессе снижения мощности реактора компенсацию реактивности производить одновременно стержнями УСП, РР и ПКАЗ, увеличивать содержание азота в продувке графитовой кладки, поддерживая в режиме снижения мощности среднюю температуру графита в активной зоне неизменной. Поддержание температуры графита неизменной в режиме снижения мощности обеспечивает невозможность дополнительного ввода отрицательной реактивности (в дополнение к переотравлению по ${ }^{135} \mathrm{Xe}$ ) за счет положительного коэффициента реактивности по температуре графита. В этом случае величина компенсации отрицательной реактивности меньше, а значит, и меньшее извлечение стержней РР и ПКАЗ, влияющих на смещение поля нейтронов в верхнюю часть активной зоны.

Таково было состояние с программным обеспечением и расчетными исследованиями НФХ РБМК-1000 накануне аварии на четвертом энергоблоке Чернобыльской АЭС.

В начале мая 1986 года в качестве причины аварии на Чернобыльской АЭС была выдвинута версия, связанная с недостатком конструкции стержней PP (концевой эффект реактивности). Однако выполнить оценку 
реальности данного предположения как первопричины аварии в тот период оказалось невозможно ввиду отсутствия полномасштабных нестационарных программ. Тогда в нескольких организациях СССР только начались их разработки и, учитывая сложность ситуации, усилия были направлены на их интенсификацию.

Для установления возможных причин аварии на Чернобыльской АЭС была создана Государственная комиссия при Госатомнадзоре СССР под руководством первого заместителя председателя Госатомнадзора СССР, члена-корреспондента АН СССР Сидоренко Виктора Алексеевича. В состав комиссии входили известные ученые: Н. Н. Пономарев-Степной, Я. В. Шевелев, А. А. Абагян и др. Одна из задач в рамках работы этой комиссии состояла в проведении оценки возможной величины концевого эффекта стержней СУЗ и его роли в развитии аварии, используя имеющиеся в различных организациях расчетные коды и библиотеки нейтронно-физических констант. Для решения этой задачи к работе комиссии были привлечены и авторы данной статьи В. В. Токаревский и В. А. Халимончук. Труд был изнуряющий. Так как для расчета одного варианта по программе DST требовалось нескольких часов при использовании самой мощной на то время ЭВМ ЕС-1061, организовать такие расчеты без фоновых задач можно было только в ночное время в киевском институте ЗНИИЭП. Ночью - расчеты, согласованные в комиссии, утром - спецавиарейсы из Борисполя в Москву и работа в Государственной комиссии по анализу причин аварии, сравнение с результатами расчетов других организаций и обсуждение последующих расчетов, вечером - обратно в Киев. На следующий день - работа в том же режиме, и так приблизительно две недели. Иногда терялась ориентация о месте нахождения: Киев, Москва? По результатам расчетов с помощью стационарных версий трехмерных программ [5, 6] была выполнена оценка величины концевого эффекта стержней СУЗ в состоянии энергоблока Чернобыльской АЭС непосредственно перед аварией в зависимости от величины оперативного запаса реактивности, аксиального распределения поля нейтронов, проведен анализ влияния ввода стержней УСП на величину этого эффекта. Расчетами было установлено, что положительный выбег реактивности мог достигнуть значения, равного эффективной доле запаздывающих нейтронов $\left(\beta_{э ф ф}\right)$ или более в зависимости от исходного значения ОЗР и исходного аксиального распределения плотности потока тепловых нейтронов. Даже при ОЗР, превышавшем регламентное значение (15 стержней РP), концевой эф-

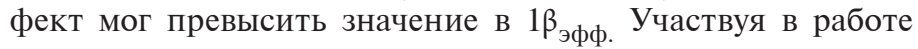
комиссии, чувствовалось, с каким переживанием случившегося и ответственностью относились все ее члены к решению поставленных задач. Результаты выполненной сотрудниками расчетной группы ИЯИ АН УССР работы были оформлены в виде отчета. Отчет для ознакомления затребовал заведующий сектором атомной энергетики при ЦК КПСС Копчинский Г. А., что и было сделано В. А. Халимончуком. В беседе с Г. А. Копчинским звучала настоятельная необходимость разобраться в причинах аварии. После ознакомления с отчетом Г. А. Копчинский попросил срочно передать его главному инженеру «Союзатомэнерго» Б. Я. Прушинскому.

На протяжении мая-декабря 1986 года максимальные усилия сосредоточились на скорейшей разработке блоков решения нестационарных уравнений диффузии в одно- и двухгрупповом приближениях и на их внедрении соответственно в программы $[5,6]$ для исследования быстрых переходных режимов эксплуатации РБМК-1000, включая отдельные проектные аварии, которые, как уже выяснилось, моделировать можно лишь при рассмотрении полномасштабной активной зоны. Здесь необходимо отметить большую позитивную роль творческого взаимодействия со старшим научным сотрудником ИАЭ им. И. В. Курчатова Краюшкиным А. В., в результате чего уже к концу 1986 года были работающие версии программ $[5,6]$ с блоками решения нестационарных уравнений диффузии. В научном плане этот период стал творческим и самым памятным: все делалось впервые, использовать внешний опыт не всегда было возможно, а зачастую он просто отсутствовал.

В дальнейшем в послеаварийный период были выполнены такие важные работы, как:

- оценка мероприятий по повышению безопасности эксплуатации РБМК-1000;

- исследование максимальной проектной аварии в различных состояниях активной зоны, с разным оперативным запасом реактивности, разными значениями парового коэффициента реактивности, с учетом модернизации стержней СУЗ и системы аварийной защиты;

- моделирование аварий, связанных с разрывом раздаточного коллектора и обезвоживанием контура СУЗ;

- исследование стержней-самоходов СУЗ с учетом системы локальной системы регулирования (ЛАР) и защиты (ЛАЗ);

- разработка алгоритма решения двухгрупповых уравнений диффузии с использованием метода межузловой нодализации [8] для программы STEPAN [9];

- технико-экономическое обоснование дожигания топлива второго энергоблока Чернобыльской АЭС в первом энергоблоке

Результаты исследования максимальной проектной аварии были включены Главным конструктором РБМК-1000 в послеаварийный ТОБ РБМК-1000 Чернобыльской АЭС.

В рамках работы по технико-экономическому обоснованию дожигания топлива второго энергоблока в первом энергоблоке был определен регламент транспортировки топлива из бассейна второго энергоблока с помощью контейнера TK-8 в бассейн первого энергоблока из условия, чтобы температура топлива при постановке в бассейн первого блока не приводила к подкипанию охлаждающей воды. Кроме того, проведена оценка экономического эффекта от дожигания топлива второго энергоблока в первом.

Позже выполнены расчеты по моделированию Чернобыльской аварии, их результаты детально представлены в [7]. Они свидетельствуют, что причиной аварии являлись недостатки конструкции ОР СУЗ и большое значение положительного парового коэффициента реактивности. Известно, что реактор РБМК был тяжело управляем, особенно на низких уровнях мощности, что связано с положительным паровым коэффициентом реактивности. Кроме того, еще до аварии на Чернобыльской АЭС, во время физических пусков первого блока Игналинской и четвертого блока Чернобыльской АЭС в 1983 году, фиксировалось введение положительной реактивности при погружении в активную зону ОР СУЗ; очень вероятно проявление концевого эффекта и во время аварии на первом энергоблоке Ленинградской АЭС в 1975 году. Несмотря на то что отдельными учеными и специалистами по физике РБМК поднимался вопрос о необходимости повышения его безопасности, до Чернобыльской аварии, к сожалению, 
ничего не было предпринято. Только на Курской АЭС в одностороннем порядке стержни УСП задействовались в аварийной защите и, как показывают результаты моделирования, этого было бы достаточно для предотвращения и Чернобыльской аварии [7].

В период становления атомной энергетики СССР многие события и исследования в области атомной энергетики были табу для общественности. Это касается аварии на первом блоке Ленинградской АЭС в 1975 году, разрыва канала на первом блоке Чернобыльской АЭС в 1982-м и разрыва канала на третьем блоке Ленинградской АЭС в 1992-м.

Казалось бы, Чернобыльская авария должна заставить всех специалистов и ученых мыслить и работать по-другому. Тем не менее, законы «политфизики» в отдельных случаях оказывались сильнее здравого смысла и после аварии. Так, по результатам выполненных в 1989 году исследований стержней-самоходов СУЗ РБМК-1000 с учетом работы системы ЛАР и ЛАЗ была подготовлена статья «Результаты исследования самоходов стержней СУЗ в реакторах РБМК$1000 »$, в которой говорилось об отсутствии эффективности работы ЛАР-ЛАЗ при самоходах некоторых периферийных ОР СУЗ. Как показали исследования, это связано, с одной стороны, с удаленностью стержня-самохода от датчиков ЛАР-ЛАЗ, из-за чего эффективность воздействия стержней ЛАР-ЛАЗ, работающих от этих датчиков, на подавление локального всплеска мощности от самохода снижается. С другой стороны, погружение этих же стержней ЛАР-ЛАЗ экранирует боковые ионизационные камеры, задействованные в аварийной защите по мощности (АЗМ), вследствие чего АЗМ не срабатывает. Согласно экспертному заключению специалистов Главного конструктора РБМК, представленная статья не может быть опубликована и является «вредной, особенно если учесть обостренную чувствительность общественности к проблемам РБМК».

Следует отметить, что игнорирование определенных факторов или их сокрытие могут обернуться экологической катастрофой, поэтому малейшая возможность руководствоваться политическими соображениями в любых научно-технических исследованиях и, особенно, в исследованиях по безопасности атомной энергетики должна быть исключена.

В заключение отметим, что Чернобыльская авария продемонстрировала исключительную важность исследований по физике реакторов, переходным и аварийным режимам их эксплуатации. Пренебрежение подобными исследованиями или невозможность их выполнения могут препятствовать выяснению скрытых дефектов конструкции реактора и его систем безопасности, приводить к нарушению нормальных условий эксплуатации РУ, возникновению проектных аварий и даже катастроф. Такие исследования невозможны без разработки современных расчетных программ для анализа безопасности поведения РУ в различных режимах эксплуатации, включая и аварийные, а также создания специальных расчетных и экспериментальных бенчмарков, позволяющих выполнить верификацию и валидацию как самих программных средств, так и библиотек нейтронно-физических констант, используемых в них. Естественно, спектр задач в атомной энергетике не ограничивается только нейтронно-физическими исследованиями. Такими же важными направлениями исследований являются, в частности, материаловедческие и прочностные, теплогидравлический анализ, радиационная безопасность, разработка концепции и стратегии развития ядерной энергетики Украины. В Украине давно назрела необходимость создания ведомственного института для решения всего комплекса задач атомной энергетики в цельном коллективе специалистов и ученых. Такие институты созданы в каждой европейской стране, где есть АЭС. Наличие подобного института должно быть экономически выгодным Украине, поскольку в значительной степени можно будет отказаться от тендерных процедур, а большую часть исследований выполнять в собственном институте при прямом финансировании.

\section{Список использованной литературы}

1. Романенко В. С. Некоторые вопросы физики РБМК / В.С.Романенко // Вопросы атомной науки и техники. - 1981. Вып. 5 (128). - С. 8-16.

2. Методика и результаты расчета нестационарных полей энерговыделения в реакторах РБМК / В. А. Халимончук, А. В. Кучин, А. В. Краюшкин, Ю. И. Лавренов. - К. : ИЯИ АН УССР, 1985. - 38 с. - (Препринт КИЯИ-85-19)

3. Программа расчета полиячейки РБМК в трехмерной геометрии с учетом обратной связи по плотности теплоносителя, температуре графитовой кладки и концентрации ксенона-135 : Отчет ИЯИ АН УССР. - К., 1983.

4. «TEGA-2» - программа расчета теплогидравлических параметров теплоносителя в технологическом канале РБМК : Отчет ИЯИ АН УССР. - К., 1977.

5. Халимончук В. А. ТРЕП - быстродействующая программа для исследования нестационарных режимов РБМК в трехмерной геометрии / В. А. Халимончук, А. В. Краюшкин. - К. : ИЯИ НАНУ, 1990. - 32 с. - (Препринт КИЯИ-90-18).

6. Марьяненко В. Д. DST - программа расчета полей энерговыделения в реакторах РБМК в трехмерной геометрии / В. Д. Марьяненко, Ю. И. Лавренов // Вопросы атомной науки и техники. Серия «Физика и техника ядерных реакторов». - 1984. Вып. 8 (45). - С. 25-29.

7. Халимончук B. A. Оценка вклада парового коэффициента реактивности и концевого эффекта СУЗ в развитие аварии на энергоблоке № 4 Чернобыльской АЭС / В. А. Халимончук, А. В. Кучин, В. В. Токаревский // Ядерна та радіаційна безпека. 2016. - № 1 (69). - C. $00-00$.

8. TaKeda T. Extension of Askew's Coarse Mesk Method to Few Group Problems for Calculating Two-Dimensional Power Distribution in Fast Breeder Reactors / TaKeda T., Komano Y. // J. of Nucl. Sci. and Techn. -1978. - V. 15. - P. 523-532.

9. Краюшкин A. B. STEPAN - трехмерная нестационарная программа расчета ядерных реакторов : Отчет ИАЭ. - 1987. Инв. № 33/801587.

\section{References}

1. Romanenko, V.S. (1981), "Some Issues of RBMK Physics" [Nekotoryie voprosy fiziki RBMK], Issues of Nuclear Science and Engineering, No. 5 (128), pp. 8-16. (Rus)

2. Khalimonchuk, V.A., Kuchin, A.V., Kraiushkin, A.V., Lavrenov, Yu.I. (1985), "Methodology and Results for Calculation of Nonstationary Energy Fields in RBMK" [Metodika i rezultaty rascheta nestatsionarnykh polei energovydeleniia v reaktorakh RBMK], Kyiv, Institute for Nuclear Research, Ukrainian SSR, 38 p. (Preprint KIYaI-85-19). (Rus)

3. "Program for Calculation of RBMK Polycell in ThreeDimensional Geometry Considering Feedback from Coolant Density, Temperature of Graphite Stack and Concentration of Xenon-135" [Programma raschiota poliiacheiki RBMK $\mathrm{v}$ trekhmernoi geometrii s uchiotom obratnoi sviazi po plotnosti teplonositelia, temperature grafitovoi kladki i kontsentratsii ksenona-135], (1983), Report of the Institute for Nuclear Research, Ukrainian SSR, Kyiv. (Rus) 
4. "TEGA-2 - Program for Calculation of Thermal and Hydraulic Parameters of the Coolant in RBMK Process Channel" [TEGA-2, programma raschiota teplogidravlicheskikh parametrov teplonositelia $\mathrm{V}$ tekhnologicheskom kanale RBMK], (1977), Report of the Institute for Nuclear Research, Ukrainian SSR, Kyiv.

5. Khalimonchuk, V.A., Kraiushkin, A.V. (1990), "TREP - FastActing Program for Research of RBMK Nonstationary Modes in Three-Dimensional Geometry" [TREP - bystrodeistvuiushchaia programma dlia issledovaniia nestatsionarnykh rezhimov RBMK v triokhmernoi geometrii], Kyiv, Institute for Nuclear Research, NASU, 32 p. (Preprint KIYaI-90-18). (Rus)

6. Marianenko, V.D., Lavrenov, Yu.I. (1984), "DST - Program for Calculation of RBMK Energy Fields in Three-Dimensional Geometry" [DST - programma raschiota polei energovydeleniia $\mathrm{v}$ reaktorakh RBMK v triokhmernoi geometrii], Issues of Nuclear Science and Engineering, Nuclear Reactor Physics and Engineering, No. 8 (45), pp. 25-29. (Rus)

7. Khalimonchuk, V.A., Kuchin, A.V., Tokarevsky, V.V. (2016), "Assessment of Contributions by Void Reactivity Coefficient and End Effect of Control Rods into ChNPP-4 Accident Progression" [Otsenka vklada parovogo koeffitsienta reaktivnosti i kontsevogo effekta SUZ v razvitiie avarii na energobloke No. 4 Chernobylskoi AES], Nuclear and Radiation Safety, No. 1 (69), pp. 00-00. (Rus)
8. TaKeda T., Komano Y. (1978), “Extension of Askew’s Coarse Mesk Method to Few Group Problems for Calculating Two-Dimensional Power Distribution in Fast Breeder Reactors", J. of Nucl. Sci. and Techn.,V. 15, pp. 523-532.

9. Kraiushkin, A.V. (1987), "STEPAN - Three-Dimensional Nonstationary Program for Nuclear Reactor Calculation” [STEPAN triokhmernaiia nestatsionarnaia programma raschiota yadernykh reaktorov], Report of the Institute of Atomic Energy, Inv. No. 33/801587. (Rus) 